\title{
Polarization squeezing by optical Faraday rotation
}

\author{
Jacob F. Sherson and Klaus Mølmer \\ Danish National Research Foundation Center for Quantum Optics \\ Department of Physics and Astronomy, University of Aarhus \\ DK 8000 Aarhus C, Denmark
}

(Dated: 15th August 2018)

\begin{abstract}
We show that it is possible to generate continuous-wave fields and pulses of polarization squeezed light by sending classical, linearly polarized laser light twice through an atomic sample which causes an optical Faraday rotation of the field polarization. We characterize the performance of the process, and we show that an appreciable degree of squeezing can be obtained under realistic physical assumptions.
\end{abstract}

PACS numbers: 03.67.-a; 32.80.Qk; 42.50.Dv

Highly squeezed states of light are valuable for numerous quantum information protocols and high precision metrology . Several techniques exist for the generation of squeezed states. In [1] the non-linear phase evolution due to the Kerr effect in an optical fiber was used to produce $5.1 \mathrm{~dB}$ of polarization squeezing. Cold atomic samples in high-finesse cavities cause similar Kerr-like effects, and $1.5 \mathrm{~dB}$ of quadrature squeezing [2] and $\sim 0.5$ $\mathrm{dB}$ of polarization squeezing 3$]$ has been observed. The most well established technique to date, however, is to use non-linear crystals in very good cavities (e.g. $\sim 7$ $\mathrm{dB}$ in [4]). In this Letter we show, however, that by reflecting a light beam so that it interacts twice through the off-resonant Faraday-type interaction with an atomic sample, a simple, robust, and efficient source of strongly squeezed light is obtained, which may well outperform the above mentioned schemes.

The optical Faraday rotation of light passing through a spin polarized atomic medium has been applied in a number of recent experiments to demonstrate entanglement and squeezing of atomic samples [5, [] ], atomic quantum memories for light [7], and teleportation of quantum states between light and matter [8]. In these experiments, the collective atomic population distribution on internal states is a quantum degree of freedom, and the measurement of the polarization of the light after the interaction alters the collective atomic quantum state. Measurements play an important role in the experiments reported in $5,66,7,[8]$, but it has also been shown that multiple interactions between a light pulse and an atomic medium 9, 10, 11, 12 suffice to effectively couple atoms and fields e.g. resulting in interspecies entanglement, atomic squeezing, or transfer of quantum states.

Our proposed physical set-up is sketched in Figure 1. In part a) we depict an atomic gas, spin polarized perpendicular to the plane of the figure, through which a cw beam or a pulse of linearly polarized light is transmitted twice from different directions. In part b) of the figure we suggest an implementation with two oppositely polarized samples which are both traversed twice by the laser beam. As will become clear below, the desired dynamics arises solely in the limit in which the light field passes through the atoms from both directions simultaneously. a)

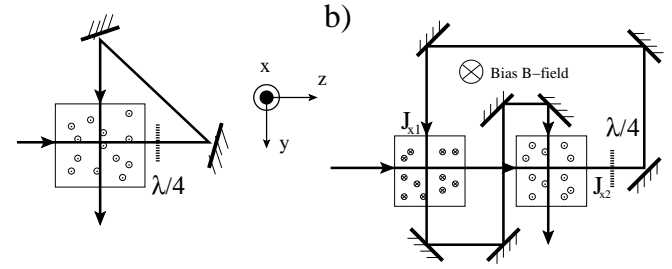

Figure 1: Physical setup for production of squeezed light. In panel a), a coherent $\mathrm{cw}$ beam or pulse of linearly polarized light is transmitted through a spin polarized atomic gas, its polarization is rotated, and it is transmitted through the gas a second time from a different direction. The output field is polarization squeezed. In panel b), two oppositely polarized gases located in a constant B-field are traversed by the light field twice. The output field is polarization squeezed at the Larmor frequency sidebands.

We treat the atomic ground state as an effective spin$1 / 2$ system and introduce the collective spin vector $\hat{\vec{J}}$. The polarization degrees of freedom of the linearly polarized optical field are accounted for by the Stokes vector $\hat{\vec{S}}$, and both of these effective spin operators obey the usual angular momentum commutator relations. As discussed in e.g. [5, 7] the Faraday interaction $\propto \hat{S}_{z} \hat{J}_{z}$ causes different phase shifts on the $\sigma^{+}$and $\sigma^{-}$circularly polarized field components due to the population difference on the atomic magnetic sub-states, equivalent to a rotation of the linear optical polarization. Conversely, the light induced AC-Stark shift imposes a phase difference between the atomic magnetic sub-states equivalent to an optical spin rotation around the z-axis proportional to the intensity difference between $\sigma^{+}$and $\sigma^{-}$field components.

For atoms which are spin polarized along the $x$-axis, it is useful to apply the Holstein-Primakoff approximation [13], where the macroscopic $x$-component of the collective spin is treated as a c-number, and where the dimensionless variables $\left(x_{a t}, p_{a t}\right)=\left(\hat{J}_{y}, \hat{J}_{z}\right) / \sqrt{J_{x}}$ obey the canonical commutator relation $\left[x_{a t}, p_{a t}\right]=i$ (here, and throughout, we set $\hbar=1)$. The minimum variances $\operatorname{Var}\left(x_{a t}\right)=$ $\operatorname{Var}\left(p_{a t}\right)=1 / 2$ reflect the binomial distribution of population on the spin-y and -z eigenstates. We also apply 
the Holstein-Primakoff approximation to the time dependent Stokes vector components representing the field at a given distance along the direction of propagation, so that the operators $\left(x_{p h}(t), p_{p h}(t)\right)=\left(S_{y}(t), \widehat{S}_{z}(t)\right) / \sqrt{S_{x}(t)}$ with dimension $1 / \sqrt{\text { time }}$, obey the canonical commutator relation $\left[x_{p h}(t), p_{p h}\left(t^{\prime}\right)\right]=i \delta\left(t-t^{\prime}\right)$.

The Faraday interaction Hamiltonian can be rewritten in the new variables, $H=\kappa p_{a t} p_{p h}[\mathbf{7},[8,9,10]$, and it is observed to be of quantum non-demolition (QND) type, i.e., it preserves the atomic variable $p_{a t}$ and the field variable $p_{p h}$. The coupling strength is $\kappa^{2}=\gamma \alpha$, with the optical depth on resonance $\alpha=N_{A} \frac{\sigma}{A}$ and the spontaneous emission rate $\gamma=\Phi \frac{\sigma}{A} \frac{\Gamma^{2}}{\Delta^{2}} . N_{A}$ is the number of atoms that interacts with the light, $\sigma$ is the resonant light scattering cross section of a single atom, $A$ is the cross section of the optical beam, $\Phi$ is the photon flux, $\Gamma$ is the natural linewidth of the atomic transition, and $\Delta$ is the detuning from the optical transition. $\kappa$ has the dimension $1 / \sqrt{\text { time }}$, and $\kappa^{2}$ will be the natural scale for the frequency dependence of our results. As illustrated in Fig. 1 we intend to pass the field around and propagate simultaneously through the gas from a different direction effectively realizing also the Hamiltonian, $H=\kappa x_{a t} x_{p h}$. Our analysis of the combined interaction becomes more involved and the full dynamics is no longer QND.

We shall apply an input-output formalism, where the field variables $x_{i}(t), p_{i}(t)$ after the first exit of the atomic sample are given in terms of the entrance variables $x_{i n}(t), p_{i n}(t)$ and the atomic variables,

$$
\begin{array}{r}
x_{i}(t)=x_{i n}(t)+\kappa p_{a t}(t) \\
p_{i}(t)=p_{i n}(t) .
\end{array}
$$

In (1) we assume that the passage of light through the gas is instantaneous, and hence the atomic and field variables can all be evaluated at the same time. The light propagates and is reflected by mirrors and is therefore subject to losses. These can effectively be modelled by a transmission coefficient $\tau$, which is less than unity, and noise terms $\rho F_{x(p)}(t)$, where $\rho^{2}=1-\tau^{2},\left[F_{x}(t), F_{p}\left(t^{\prime}\right)\right]=$ $i \delta\left(t-t^{\prime}\right)$ and $\left[F_{x}(t), F_{x}\left(t^{\prime}\right)\right]=\left[F_{p}(t), F_{p}\left(t^{\prime}\right)\right]=0$. We thus modify (11) to represent the fields prior to the second passage of the gas,

$$
\begin{array}{r}
x_{i}^{\prime}(t)=\tau\left(x_{i n}(t)+\kappa p_{a t}(t)\right)+\rho F_{x}(t) \\
p_{i}^{\prime}(t)=\tau p_{i n}(t)+\rho F_{p}(t) .
\end{array}
$$

In this set of equations, we have ignored the time of propagation between the two passes. This approximation amounts to the assumption that the atomic state changes only little on the time scale of the optical propagation, which will always be the case with realistic physical parameters.

Before the second interaction between the field and the atoms the field polarization is rotated $90^{\circ}$ (using a $\lambda / 4$-plate), and the atomic sample is approached from a different direction, altogether realizing the Hamiltonian $H=\tau \kappa x_{a t} x_{p h}$, where the $\tau$ factor accounts for the reduced interaction strength because of the photon loss $\left(\Phi \rightarrow \tau^{2} \Phi\right)$ between the passages. We now express the output fields $x_{\text {out }}(t), p_{\text {out }}(t)$ in terms of the atomic variables and the intermittent field components (2):

$$
\begin{array}{r}
x_{\text {out }}(t)=x_{i}^{\prime}(t) \\
p_{\text {out }}(t)=p_{i}^{\prime}(t)-\kappa \tau x_{\text {at }}(t),
\end{array}
$$

For mathematical convenience we have applied another $90^{\circ}$ rotation of the light after the second passage.

The atoms undergo dissipation, both due to the weak excitation by the optical fields, which causes a small spontaneous emission rate $\gamma$, and because we may implement optical pumping with a rate $\gamma_{p}$ to retain the atomic macroscopic polarization along the $\mathrm{x}$-axis. The mean spin will thus obey $\frac{d J_{x}}{d t}=-\gamma J_{x}+\gamma_{p}\left(N / 2-J_{x}\right)$ with a macroscopic steady state value $J_{x}=\frac{\gamma_{p}}{\gamma+\gamma_{p}} N / 2$. The atomic variables $x_{a t}$ and $p_{a t}$ decay with the rate $\gamma_{1}=\gamma+\gamma_{p}$. The decay is accompanied by noise terms $\sqrt{2 \gamma_{2}} G_{x(p)}(t)$, where $G_{x(p)}(t)$ have the same commutator properties as the $F_{x(p)}(t)$ operators introduced for the field losses. Due to the division by $\sqrt{J_{x}}$ in the Holstein-Primakoff approximation, we obtain the value $\gamma_{2}=\gamma_{1} /\left(\frac{\gamma_{p}}{\gamma+\gamma_{p}}\right)=\left(\gamma+\gamma_{p}\right)^{2} / \gamma_{p}$ for the strength of the noise term. If there are other decoherence mechanisms present, $\gamma_{1}$ and $\gamma_{2}$ of course have to be modified correspondingly.

The atomic interaction with the field at the two passages and the damping are described by the equations

$$
\begin{gathered}
\frac{d}{d t} x_{a t}(t)=\kappa p_{i n}(t)-\gamma_{1} x_{a t}(t)+\sqrt{2 \gamma_{2}} G_{x}(t) \\
\frac{d}{d t} p_{a t}(t)=-\kappa \tau x_{i}^{\prime}(t)-\gamma_{1} p_{a t}(t)+\sqrt{2 \gamma_{2}} G_{p}(t),
\end{gathered}
$$

which have to be solved together with the field equations (23).

To solve the equations under $\mathrm{cw}$ operation, we change to the frequency domain, letting $h(t)=\frac{1}{\sqrt{2 \pi}} \int e^{i \omega t} h(\omega) d \omega$ for all operators $h=x_{a t}, p_{a t}, x_{i n}, p_{\text {in }}, x_{i}, p_{i}, x_{\text {out }}, p_{\text {out }}, F_{x}, F_{p}, G_{x}, G_{p}$. This transformation changes the argument from time to frequency in the field equations (23), and it changes the differential equations (44) into the algebraic equations

$$
\begin{gathered}
i \omega x_{a t}(\omega)=\kappa p_{i n}(\omega)-\gamma_{1} x_{a t}(\omega)+\sqrt{2 \gamma_{2}} G_{x}(\omega) \\
i \omega p_{a t}(\omega)=-\kappa \tau x_{i}^{\prime}(\omega)-\gamma_{1} p_{a t}(\omega)+\sqrt{2 \gamma_{2}} G_{p}(\omega) .
\end{gathered}
$$

We can now systematically express both the atomic variables and the output field variables in terms of the input field and noise operators, and we find by elementary operations,

$$
x_{\text {out }}(\omega)=\frac{\left(\gamma_{1}+i \omega\right)\left(\tau x_{i n}+\rho F_{x}(\omega)\right)+\tau \sqrt{2 \gamma_{2}} \kappa G_{p}(\omega)}{\gamma_{1}+i \omega+\tau^{2} \kappa^{2}} .
$$

If we assume the vacuum values for the noise power spectrum of the incident classical field and the noise variables 


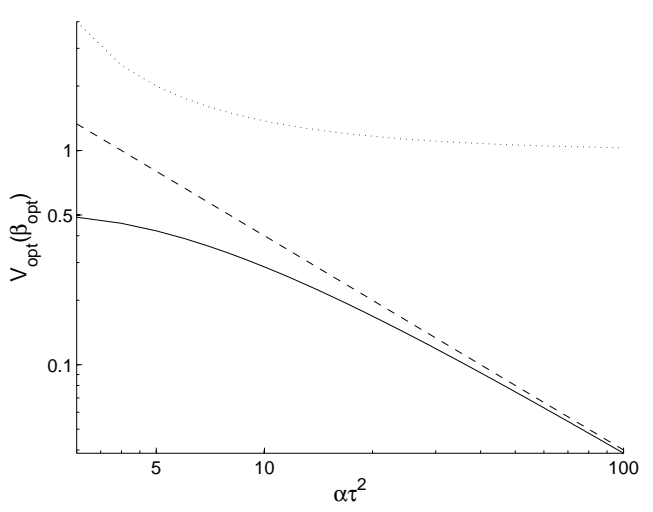

Figure 2: The optimal degree of squeezing $V_{\text {opt }}$, Eq. 9) (solid line) approached asymptotically by the expression $4 /\left(\alpha \tau^{2}\right)$ (dashed line) as functions of $\alpha \tau^{2}$. Both curves assume the optimal strength of the optical pumping, $\beta=\gamma_{p} / \gamma$, which is shown as the upper dottted line.

$\left\langle h(t) h\left(t^{\prime}\right)\right\rangle=\frac{1}{2} \delta\left(t-t^{\prime}\right) \Rightarrow\left\langle h(\omega) h\left(\omega^{\prime}\right)=\frac{1}{2} \delta\left(\omega+\omega^{\prime}\right)\right.$, the noise spectrum of squeezing of the field is given by the expectation value $\left\langle x_{\text {out }}(\omega) x_{\text {out }}\left(\omega^{\prime}\right)\right\rangle=V_{x}(\omega) \delta\left(\omega+\omega^{\prime}\right)$, and we obtain directly from (6),

$$
V_{x}(\omega)=\frac{1}{2}\left(1-\frac{2\left(\gamma_{1}-\gamma_{2}\right) \kappa^{2} \tau^{2}+\kappa^{4} \tau^{4}}{\left(\gamma_{1}+\kappa^{2} \tau^{2}\right)^{2}+\omega^{2}}\right) .
$$

The squeezing spectrum is a Lorentzian, with a width given by the atomic decoherence rate, the coupling strength to the atoms and the transmission efficiency of light between the two passages of the gas. Interestingly, the effect of losses between the two light passages of the gas only has the effect to modify the value of the interaction strength, $\tilde{\kappa}=\kappa \tau$.

Assuming that the dominant atomic decoherence mechanism is light absorption, and writing $\kappa^{2}=\gamma \alpha$ and $\gamma_{p}=\beta \gamma$, the maximum squeezing (at $\left.\omega=0\right)$ is:

$$
V_{x}(0)=\frac{1}{2} \frac{(1+\beta)^{2}\left(\beta+2 \alpha \tau^{2}\right)}{\beta\left(1+\beta+\alpha \tau^{2}\right)^{2}},
$$

Optimizing Eq. (8), we get

$$
V_{\mathrm{opt}}=\frac{1}{2} \frac{\left(2 \alpha \tau^{2}-1\right)^{3}}{\left(\alpha \tau^{2}-1\right)\left(1+\alpha \tau^{2}\right)^{3}} \rightarrow \frac{4}{\alpha \tau^{2}} \quad \alpha \tau^{2} \gg 1
$$

for $\beta=\frac{1+\alpha \tau^{2}}{\alpha \tau^{2}-2}$. In Fig. 22 we plot Eq. (9) along with the optimal value for $\beta$. As can be seen, degrees of squeezing of the order of $10 \mathrm{~dB}$ should realistically be achieved for optical depths $\alpha \approx 100$ which is routinely attained in cold atomic samples as in [6]. In a BEC optical densities of up to $10^{3}$ can be achieved potentially paving the way for unprecedented degrees of squeezing. The high degrees of squeezing are attained while the mean spin $J_{x}=\beta /(1+\beta) \cdot N / 2$ is reduced by a factor on order unity compared to its maximal value. For $\alpha \tau^{2} \gg \beta \gg 1$ the mean spin is only reduced slightly and the non-optimal

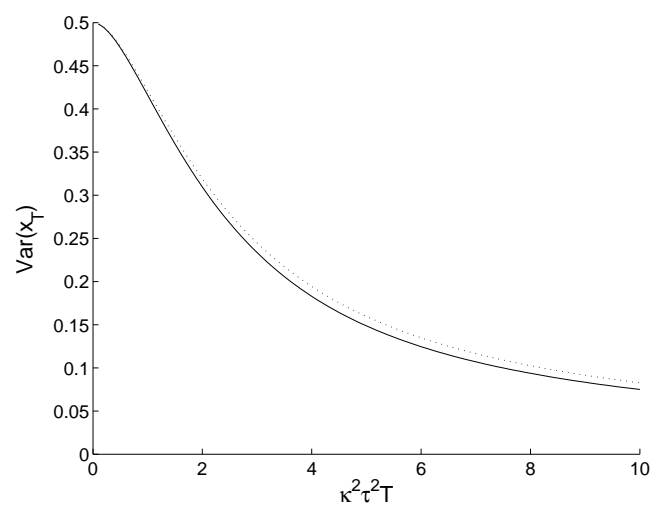

Figure 3: The variance $\operatorname{Var}\left(x_{T}\right)$ after passage of a square pulse through the single cell set-up (see Fig. 1 a)) The variance is shown as a function of $\kappa^{2} \tau^{2} T$ with finite damping $\gamma T=0.1$ (dotted line) and with no damping (solid line).

degree of squeezing approaches $\beta /\left(\alpha \tau^{2}\right)$, which can still be significant.

The atoms can also be optically pumped into the macroscopically polarized state and interact only with a finite duration pulse of light, so that the reduction in the mean spin, with rate $\gamma$, is small. The equations (24), with $\gamma_{1}=\gamma_{2}=\gamma$ can be solved directly in the time domain, expressing the time dependent output fields as integrals over the incident field and noise operators. At this point, there are rich possibilities to vary the incident field envelope and the mode function identified with the output field with the purpose to optimize the squeezing. For simplicity, we shall here assume an incident square pulse, and we shall consider the amount of squeezing in a mode defined by the same pulse envelope, i.e., we consider the single mode field operators $q_{T}=\frac{1}{\sqrt{T}} \int_{0}^{T} q_{\text {out }}(t) d t$, where $q=x$ or $p$, and where $T$ is the duration of the light pulse. From our full time dependent solution we obtain the output variances:

$$
\operatorname{Var}\left(q_{T}\right)=\frac{1}{2}\left(1 \mp \frac{\kappa^{4} \tau_{q}^{2}}{2 \gamma_{q}^{3} T}\left(2 \gamma_{q} T+1-\left(2-e^{-\gamma_{q} T}\right)^{2}\right)\right),
$$

where $q=x, p, \tau_{x}=\tau^{2}, \tau_{p}=\tau, \gamma_{x}=\gamma+\kappa^{2} \tau^{2}, \gamma_{p}=\gamma$, and where the $-(+)$ sign apply to the $x_{T}\left(p_{T}\right)$ component. In Fig. 3 we plot the attainable degree of squeezing as a function of the dimensionless quantity $\kappa^{2} \tau^{2} T$. The assumption of this calculation is that the mean spin is preserved, and we show in the figure the results for finite damping $\gamma T=0.1$ and for no damping $\gamma T=0$, which provide quite equivalent results. The attainable degree of sqeezing is clearly sizable. In the limit of no damping, Eq. (10) approaches the simple expressions

$$
\operatorname{Var}\left(x_{T}\right)=\frac{3+e^{-2 \hat{\kappa}^{2}}-4 e^{-\hat{\kappa}^{2}}}{4 \hat{\kappa}^{2}}, \quad \operatorname{Var}\left(p_{T}\right)=\frac{1}{2}+\frac{\hat{\kappa}^{4}}{6},
$$

where $\hat{\kappa}^{2}=\kappa^{2} T$. We note that the product of the two 


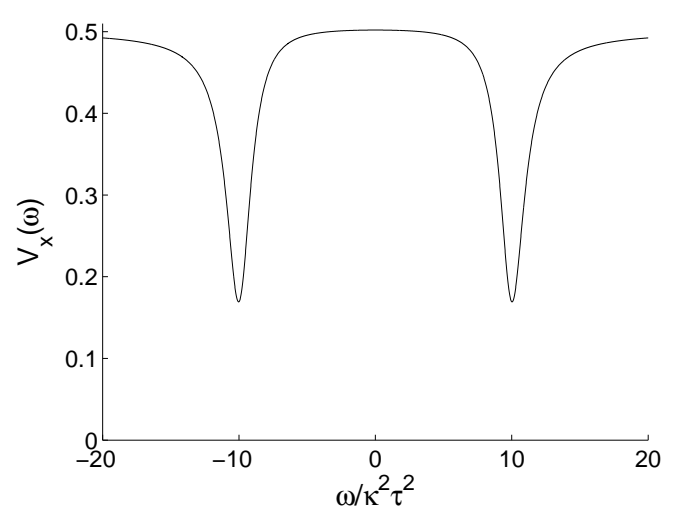

Figure 4: Squeezing spectrum for continuous wave field transmitted twice through two atomic ensembles which are larmnor precessing at freqency $\Omega$. The parameters in the figure are $\Omega=10 \tilde{\kappa}^{2}, \gamma_{1}=0.1 \tilde{\kappa}^{2}$, and $\gamma_{2}=0.2 \tilde{\kappa}^{2}$ (corresponding to $\left.\gamma=\gamma_{p}\right)$.

variance is $1 / 4$ for $T=0$ but the squeezing of the $x$ component is accompanied by a growth of the uncertainty product for $\kappa^{2} T \gg 1$.

Our proposal can also be implemented with two oppositely oriented atomic samples and a homogeneous magnetic field as illustrated in Fig. 1 b). To model this we introduce atomic variables $p_{a t, i}=J_{z, i} / \sqrt{J_{x}}$, and $x_{a t, i}= \pm J_{y, i} / \sqrt{J_{x}}$, where $i=1,2$. This gives equations of motion very similar to equations (24) and the Larmor precession caused by the constant magnetic field is modelled by adding terms $\pm \Omega x_{a t, i}$ and $\mp \Omega p_{a t, i}$ to the differential equations for $p_{a t, i}$ and $x_{a t, i}$ respectively. Note that the rotation is in opposite directions for the two atomic samples. Solving the coupled equations we get

$V_{x}(\omega)=\frac{1}{2}\left(1-\frac{4 \tilde{\kappa}^{4}\left(\gamma_{1}^{2}+\omega^{2}\right)+4 \tilde{\kappa}^{2}\left(\gamma_{1}-\gamma_{2}\right)\left(\gamma_{1}^{2}+\omega^{2}+\Omega^{2}\right)}{\gamma_{1}^{2} A^{2}+2 A \gamma_{1}\left(\omega^{2}+\Omega^{2}\right)+\left(\omega^{2}-\Omega^{2}\right)^{2}+4 \tilde{\kappa}^{4} \omega^{2}}\right)$

where $\tilde{\kappa}=\kappa \tau$ and $A=\gamma_{1}+2 \tilde{\kappa}^{2}$. Again losses between the two passages merely modify the interaction strength by a factor as was also the case in the single cell implementation (Eq. (7)). In the limit of fast rotations $(\Omega \gg \gamma, \tilde{\kappa})$ we get two well separated peaks in the squeezing spectrum centered around $\omega= \pm \Omega$ as shown in Fig.
4. Introducing $\omega= \pm \Omega+\delta$ and letting $|\Omega| \gg \delta, \gamma_{1}$, Eq. (12) reduces to a Lorentzian dependence on the frequency off-set with respect to $\pm \Omega$ with the same parameters as Eq. (7) for a single sample without rotations. It has previously been shown that the Faraday-QND interaction of a single sample without a contant bias magnetic field is regained for two oppositely oriented samples in the presence of a bias field [5, 7]. Our result shows that this correspondence extends to our non-QND situation, when the dynamics is fast compared to the magnetic precession frequency.

In summary, we have shown that both light pulses and cw light fields passing twice through an atomic gas or through two atomic samples become significantly squeezed. The width of the sqeezing spectrum is governed by the coupling strength $\kappa^{2}$, and the optimum of squeezing is controlled by the resonant optical depth. Realistic estimates for these parameters in a number of current experiments suggest that the attainable squeezing competes well with the achievements of other schemes. Optical transmission losses after the last atomic interaction can readily be modelled with a transmission coeffecient and a noise term as in Eq.(2), and the relevant single mode or continuous wave variances $V$ are modified according to, $V \rightarrow \tilde{\tau}^{2} V+\tilde{\rho}^{2} \frac{1}{2}$.

Let us conclude with a brief discussion of the "simultaneous passage" mechanism behind the squeezing. By inserting Eq. (2) into Eq. (4) it is clear that both during our cw and our longer pulse transmission, $p_{a t}$ is fed back onto itself with a negative factor, which leads to a damping of the corresponding variance. In Eq. (1), $p_{a t}$ is, in turn, mapped onto $x_{i}$ and hence onto $x_{\text {out }}$, c.f. Eqs.(23), which gives rise to the squeezed output state of light. For pulses short enough to allow a complete passage in one 'direction before the passage in the other one, it is the initial unsqueezed $p_{a t}$ which maps onto the field variable in the first interaction, and $x_{\text {out }}$ will thus be even more noisy than in the input state. The simultaneous interaction assumes that the travel time for the light between passes should be much smaller than the pulse length or the reciprocal of the $\mathrm{cw}$ squeezing bandwidth, which is readily fulfilled in experiments.
[1] J. Heersink, V. Josse, G. Leuchs, and U. L. Andersen, Optics Letters 30, 1192 (2005).

[2] A. Lambrecht, T. Coudreau, A. M. Steinberg, and E. Giacobino, Europhys. Lett. 36, 93 (1996).

[3] V. Josse, A. Dantan, L. Vernac, A. Bramati, M. Pinard, and E. Giacobino, Phys Rev. Lett. 91, 103601 (2003).

[4] S. Suzuki, H. Yonezawa, F. Kannari, M. Sasaki, and A. Furusawa, quant-ph/0602036.

[5] B. Julsgaard, A. Kozhekin, and E. S. Polzik, Nature 413, 400 (2001).

[6] J. Geremia, J. Stockton, and H. Mabuchi, Science 304, 270 (2004).
[7] B. Julsgaard, J. Sherson, J. Cirac, J. Fiurášek, and E. Polzik, Nature 432, 482 (2004).

[8] J. Sherson, H. Krauter, R. K. Olsson, B. Julsgaard, K. Hammerer, I. Cirac, and E. S. Polzik, quantph/0605095.

[9] K. Hammerer, K. Mølmer, E. S. Polzik, and J. I. Cirac, Phys. Rev. A 70, 044304 (2004).

[10] J. Sherson, A. S. Sørensen, J. Fiurasek, K. Mølmer, and E. Polzik, quant-ph/0505170.

[11] J. Fiurasek, J. Sherson, T. Opatrny, and E. Polzik, Phys. Rev. A 73, 022331 (2006).

[12] C. A. Muschik, K. Hammerer, E. S. Polzik, and J. I. 
Cirac, quant-ph/0512226.

[13] T. Holstein and H. Primakoff, Phys. Review 58, 1098

(1940). 